\title{
The Generalizability of Subliminal Mere Exposure Effects: Influence of Stimuli Perceived Without Awareness on Social Behavior
}

\author{
Robert F. Bornstein \\ Gettysburg College
}

\author{
Dean R. Leone and Donna J. Galley \\ State University of New York at Buffalo
}

\begin{abstract}
This article describes three experiments investigating the extent to which subliminal mere exposure effects are obtainable not only with simple stimuli but also with complex human stimuli in social situations. In the first experiment, undergraduate subjects were exposed to slides of abstract geometric figures at both subliminal (i.e., $4 \mathrm{~ms}$ ) and supraliminal exposure durations. Subjects' attitudes toward the subliminally presented stimuli became significantly more positive with repeated exposures, even when subjects were unaware that exposures had occurred. Experiment 2 demonstrated that similar attitude changes are produced by subliminal exposure to photographs of actual persons. The results of Experiment 3 indicate that attitudes toward persons encountered in the natural environment of the psychology experiment are also enhanced by subliminal exposure to a photograph of that person. These findings are discussed in the context of prevailing models of mere exposure effects as well as an alternative psychodynamic model. Implications for research on attitudes, social cognition, and interpersonal object choice are also discussed.
\end{abstract}

The investigation of subliminal and marginal phenomena represents an interface among the fields of personality, social, and cognitive psychology. Although the empirical study of these phenomena dates from early in this century (Poetzl, 1917) and is one of the few areas of research cited by Freud (1900/1919), recent interest in this area was stimulated by controversial studies conducted during the 1950 s that purported to demonstrate that subliminal exposure to certain verbal messages influenced consumer behavior (McConnell, Cutler, \& McNeil, 1958). Although these early studies have generally proved to be methodologically flawed and irreplicable, interest in the extent to which stimuli perceived without awareness can influence cognition, affect, and behavior has continued to the present. Within social psychology, researchers continue to examine the extent to which such stimuli can influence attitudes and behavior toward a variety of stimuli, including consumer products, political and social issues, and other people (Nisbett \& Wilson, 1977). Clinical psychologists have investigated the extent to which exposure to subliminal stimuli has different effects on individuals with different personality types and psychopathologies and have also studied the degree to which "drive-related" stimulus content affects ease (and difficulty) of perception (Silverman, 1983). Stimulated by the development of signal-detection theory

\footnotetext{
We would like to thank Gail Bruder, Paul R. D'Agostino, Edwin P. Hollander, Edward S. Katkin, Mary A. Languirand, Richard M. O'Neill, and especially Joseph M. Masling for helpful comments on earlier versions of this article.

Portions of this article were presented at the April 19, 1985, meeting of the Psychological Association of Western New York, Williamsville, and at the April 15, 1986 meeting of the Eastern Psychological Association, New York.

Correspondence concerning this article should be addressed to Robert F. Bornstein, Department of Psychology, Gettysburg College, Gettysburg, Pennsylvania 17325.
}

(Green \& Swets, 1966) and information processing models of selective attention (Triesman, 1969), cognitive (as well as social) psychologists have taken an increasing interest in issues related to the perception of marginal and subliminal stimuli and have used methodologies from signal-detection theory to study such questions empirically. In recent years there has been a growing interest in developing and validating neuroanatomical models to explain biological mechanisms underlying the perception and processing of subliminal and marginal phenomena (e.g., Dixon, 1981; Winson, 1984).

Psychologists studying Zajonc's well-known mere exposure effect (Zajonc, 1968) have conducted several studies using subliminal stimuli to investigate the extent to which stimulus awareness is a prerequisite for the production of these effects. Kunst-Wilson and Zajonc (1980) used the mere exposure paradigm to demonstrate that significant, measurable attitude changes were obtainable with exposure durations as brief as 1 ms; this work has been replicated and extended in a series of studies by Seamon and his colleagues (Seamon, Brody, \& Kauff, 1983a, 1983b; Seamon, Marsh, \& Brody, 1984). However, although Seamon et al. and Kunst-Wilson and Zajonc have consistently demonstrated that conscious awareness of stimuli is not a prerequisite for the production of measurable attitude change, the stimuli used in these studies-irregular geometric figures (polygons) - are far removed from those generally encountered in the course of daily life. Most experimental psychologists have used simple, neutral stimuli in studies of subliminal phenomena to avoid methodological confounds such as prior familiarity with, and idiosyncratic associations to, particular stimuli. Thus, geometric figures, nonsense syllables, and abstract visual symbols are frequently used as stimuli in such studies. For social and clinical psychologists, however, the primary object-or environmental stimulus-worthy of study is other people, and no studies of subliminal perception have used people as the target (i.e., subliminally exposed) stimulus and 
then examined attitudes toward these people. The subliminal mere exposure paradigm affords a ready means of examining the extent to which subliminal and marginal phenomena influence social cognitions and attitudes toward unfamiliar people.

This article consists of a series of three experiments that range from a laboratory study of subliminal perception (Experiment 1) to an investigation of subliminal mere exposure effects in vivo (Experiment 3). These experiments investigate the extent to which subliminal mere exposure effects are obtainable under conditions in which subjects make judgments regarding complex, whole individuals rather than words or pictures. Before attempting to investigate subliminal effects in vivo, however, a replication of Kunst-Wilson and Zajonc's (1980) findings regarding subliminal exposure and affective preference for abstract visual stimuli was warranted.

Experiment 1 is a modified replication of Kunst-Wilson and Zajonc's (1980) experiment, as revised by Seamon et al. (1984). Subjects were asked to make forced-choice affect and recognition judgments for pairs of irregular polygons, following subliminal or supraliminal exposure to one member of each polygon pair. Experiment 2 is similar in procedure to Experiment 1 except that the stimuli consisted of photographs of normal adults, with subjects asked to make forced choice affect and recognition judgments following subliminal or supraliminal exposure to the photograph stimuli. Experiment 3 investigates the extent to which subliminal exposure influences subjects' perceptions of and interactions with other people in a social situation.

\section{Experiment 1-Zajonc's Subliminal Mere Exposure Effect: A Modified Replication}

To investigate the effects of exposure duration on affect and recognition judgments of simple polygon stimuli, Seamon et al. (1984) exposed subjects to five repetitions each of 10 irregular polygons at exposure durations ranging from 0 to $48 \mathrm{~ms}$. They found that at exposure durations of less than $12 \mathrm{~ms}$, affect judgments were significantly better than recognition judgments in distinguishing previously seen from novel stimuli, whereas at exposure durations of $12 \mathrm{~ms}$ or greater, the accuracy of recognition judgments in selecting previously exposed stimuli surpassed that of affect judgments. In this study, we attempted to replicate these findings with the following methodological changes.

1. Subjects were tested individually, not in groups, to ensure maximum subject investment in the study.

2. Rather than first exposing subjects to all of the polygon stimuli and then collecting affect and recognition judgments (as was the procedure in the Seamon et al. study), stimuli were presented one at a time (five exposures per stimulus), and judgments were made immediately following stimulus exposure. This procedural change was made to minimize the latency between stimulus exposure and judgment by the subject, as recommended by Silverman (1977).

3. Rather than following each stimulus exposure with a 30ms bright energy field mask, as was done by Seamon et al., we used a 4-s prestimulus energy field mask that went off as the stimulus was exposed. Previous research has shown that this type of mask will yield significant behavioral effects while nei- ther recall, recognition, or discrimination judgments made for stimuli presented under these conditions exceed chance accuracy (Masling, Bornstein, Poynton, Reed, \& Katkin, 1987).

4. We presented stimuli with a Scientific Prototype threefield tachistoscope, rather than a slide projector equipped with a camera shutter, to ensure that subjects were attending to the stimulus rather than focusing their vision elsewhere.

5. Affect and recognition judgments were made by using polygon stimulus cards (see the Method section of this study for a fuller description of these stimuli) rather than stimuli projected onto a screen.

Aside from these changes, the experiment was designed to replicate the procedures of Seamon et al. (1984). For the purpose of efficiency, only two exposure durations were used, rather than the six exposure durations that Seamon et al. used. Subjects were exposed either subliminally (i.e., for $4 \mathrm{~ms}$ ) or supraliminally (for $48 \mathrm{~ms}$ ) to individual polygon stimuli. Forcedchoice affect and recognition judgments were then made between the target and a matched polygon stimulus. The dependent measure used was percentage of previously exposed stimulus selection. Following Kunst-Wilson and Zajonc (1980), we hypothesized that subjects would have "clear preferences for exposed stimuli even though subjects in a recognition memory task can not discriminate them from novel stimuli" (p. 557).

As an additional test of whether perception of stimuli in the subliminal (4-ms) condition actually took place without subjects' conscious awareness, a discrimination task similar to that described by Silverman (1966) was introduced. A second group of 10 subjects were asked to make judgments regarding the presence or absence of polygon stimuli for a series of 60 subliminally presented slides, half of which were stimulus slides and half of which were blanks. If perception of polygon stimuli in the 4ms condition actually took place without conscious awareness, then accuracy of target selection in the stimulus discrimination task should not differ significantly from chance (i.e., 50\%) accuracy.

\section{Method}

Subjects. Subjects were 62 undergraduate students ( 26 male and 36 female) enrolled in introductory psychology classes at the State University of New York (SUNY) at Buffalo. An additional 10 undergraduates (7 male and 3 female) who did not participate in other aspects of this study were used in the discrimination task.

Materials and apparatus. Stimuli were 20 irregular polygons selected from those of Vanderplas and Garvin (1959), the same stimulus source used by Seamon et al. (1983a, 1983b, 1984). Polygons of comparable complexity and attractiveness were paired, yielding three pairs of foursided polygons, four pairs of six-sided polygons, and three pairs of eightsided polygons. Each stimulus consisted of a black construction-paper polygon glued to a white $8 \times 10$ in. cardboard card. Each polygon occupied approximately the same amount of space (i.e., $20 \%-25 \%$ ) on the stimulus card.

Slide stimuli were constructed by photographing each polygon card as a negative slide. The polygon occupied $10 \%-15 \%$ of the total area of each slide. Stimuli were projected through a Scientific Prototype threefield tachistoscope with exposure duration, background field luminescence, and target field luminescence preset by an experimenter who had no contact with the subjects. The experimenter who ran subjects was blind to all information regarding exposure duration.

Procedure. Subjects were told that they were participating in a study 
of how rapidly people are able to process visual information. They were informed that they would be shown slides of geometric figures and asked to make a forced-choice preference judgment between two polygon stimulus cards showing similar figures. Subjects were then shown five repetitions of one member of the first polygon pair at either $4 \mathrm{~ms}$ or $\mathbf{4 8}$ ms. Exposures were $5 \mathrm{~s}$ apart, each exposure preceeded by a 4-s bright energy field mask with a focus dot in the center; the polygon stimulus was presented in the same position as the focus dot immediately after the blank field was turned off. The illumination of the blank field was $15 \mathrm{fl}$, and that of the stimulus field was, on average, $8 \mathrm{fl}$.

Following five exposures to one member of a given polygon pair, subjects were presented with both stimulus cards from that pair and asked to indicate which they preferred. This procedure was repeated for each of the 10 polygon stimulus pairs; choices made under these conditions will hereinafter be referred to as affect judgments.

Following this (the affect phase of the experiment), subjects were put through a similar procedure, with two important differences. The first was that subjects were asked during this phase of the experiment (the recognition phase) to try to choose which member of each pair they had just seen, not which higure they preferred. Subjects were also asked to make a confidence rating for each recognition response, as described by Kunst-Wilson and Zajonc (1980), rating each response as either sure, half sure, or guess. The second difference was that although one member of each of the same 10 polygon stimulus pairs was again presented, the selection of individual slides was random; sometimes the subject was shown the same stimulus as had been shown on the first trial and sometimes was shown its pair polygon. Subjects were informed of this at the start of the second phase of the experiment.

For each subject, then, we collected 10 affect judgments, 10 recognition judgments, and 10 confidence ratings (one for each recognition response). After all data had been collected, subjects were debriefed and told of the purposes of the experiment.

Discrimination lask. Ten additional subjects ( 7 male and 3 female), who had not participated in earlier phases of the experiment, attempted to discriminate stimulus slides from blank slides under 4-ms exposure conditions. Subjects were shown a series of 60 slides presented 5 times each, at 4-ms exposure durations, with each slide preceeded by a 4-s bright energy field mask; half of the slides contained polygon stimuli and half were blank slides. Each subject was informed of this prior to the start of the procedure. The subject was instructed to report, immediately following five repetitions of a given slide, whether the slide was a polygon stimulus or a blank.

\section{Results}

This experiment used a mixed design in which exposure duration ( $4 \mathrm{~ms}$ vs. $48 \mathrm{~ms}$ ) was a between-subjects variable and judgment type (affect vs. recognition) was a within-subjects variable. Percentages of previously seen polygon selection as a function of judgment type and exposure duration is presented in Figure 1. In the 4-ms condition, percentages of previously seen stimuli selected in the affect and recognition conditions were $57.8 \%$ and $50.1 \%$, respectively; at $48 \mathrm{~ms}$, these percentages were $56.3 \%$ and $94.8 \%$.

Analysis of variance showed significant main effects of judgment type, $F(1,60)=31.66, p=.001$, and exposure duration, $F(1,60)=65.55, p=.001$, and a significant interaction between these variables, $F(1,60)=87.56, p=.001$. A $t$ test comparing subjects' affect and recognition judgments in the 4-ms condition showed that affect judgments were significantly more accurate than recognition judgments at this exposure duration, $\imath(74)=12.16, p<.001$. Affect judgments in both the 4-ms and 48-ms conditions were significantly more accurate in selecting

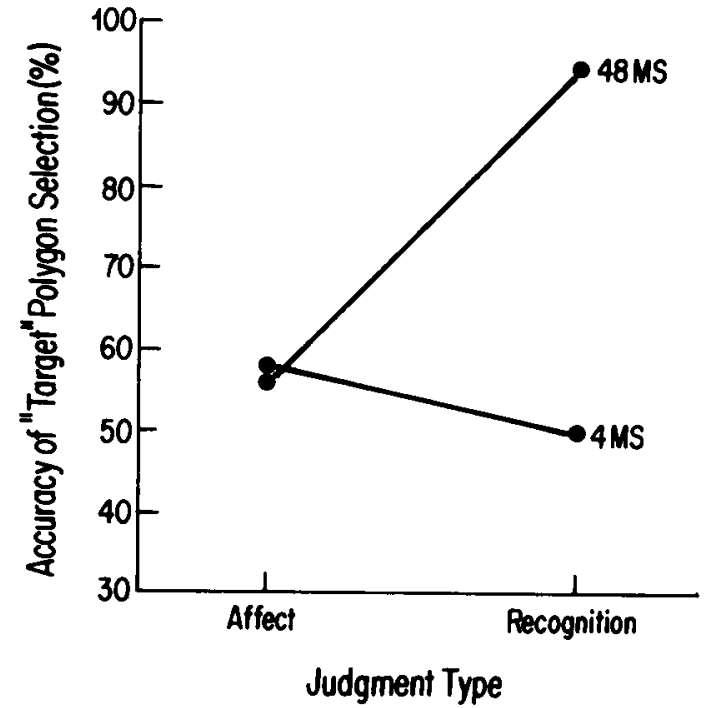

Figure 1. Percentage of target (i.e., previously seen) polygon selection as a function of exposure duration (4 ms vs. $48 \mathrm{~ms}$ ) and judgment type (affect vs. recognition).

previously exposed stimuli than would be expected by chance, $\chi^{2}(1, N=38)=8.12, p<.01$, for $4-m s$ exposures and $\chi^{2}(1, N=$ 24) $=9.81, p<.01$, for 48 -ms exposures.

Subjects' mean confidence ratings (in which a low score reflects greater confidence) for recognition judgments made in the 4-ms and 48-ms conditions were $2.51(S D=0.48)$ and 1.18 $(S D=0.28)$, respectively, as would be expected. Accuracy of recognition judgments did not correlate with subjects' mean confidence rating in the $4-\mathrm{ms}$ condition $(r=.12, n s)$ or in the 48-ms condition $(r=-.20, n s)$.

The mean number of slides correctly identified by subjects in the discrimination task was $31.4(52 \%)$. Overall, subjects correctly identified 314 of 600 slides, which does not differ signifcantly from chance accuracy, $\chi^{2}(1, N=600)=1.30$. The number of correct identifications ranged from $27(45 \%)$ to $34(57 \%)$.

\section{Discussion}

These results suggest that subjects' preferences for abstract geometric shapes may be significantly influenced by subliminal presentation of these stimuli, using exposure durations so brief that subjects are unable to recognize the polygon figures and are in fact unaware of having been exposed to any stimuli at all. These results confirm the findings of Kunst-Wilson and Zajonc (1980) and Seamon et al. (1984) and demonstrate that "subjects can select previously shown target stimuli on the basis of affective preference even when they fail to do so by recognition memory" (Seamon et al., 1984, p. 468). Our results (as well as those of Kunst-Wilson and Zajonc and Seamon et al.) are also consistent with the psychoanalytic notion that stimuli perceived without awareness may have significant effects on subsequent cognitions, affects, and behaviors (Bornstein \& Masling, 1984; Silverman, 1983). Our findings represent an extension of Seamon et al.'s work in that additional control measures were used to ensure that perception of the subliminal stimuli actually took 
place without awareness. That this experiment obtained results consistent with those reported by Kunst-Wilson and Zajonc (1980) and Seamon et al. (1984), using a significantly modified methodology, attests to the robustness of the subliminal mere exposure effect. Now three independent laboratories using three different methodologies have obtained consistent results on three separate subject populations.

Supporting the contention that perception of the 4-ms stimuli took place without conscious awareness, confidence ratings did not correlate with accuracy of recognition judgment in either the 4-ms or the 48-ms condition. In the $48-\mathrm{ms}$ condition, the absence of a significant correlation is probably due to a ceiling effect; all but one subject in this condition correctly recognized $90 \%-100 \%$ of the target stimuli. However, because subjects in the 4-ms condition were unable to determine the accuracy of their recognition judgments at this exposure duration (i.e., because they were unable to discriminate between correct and incorrect choices), this experiment provides additional evidence that stimuli perceived without awareness can result in significant changes in attitude.

\section{Experiment 2-Mere Exposure of Complex Stimuli: Affect Preferences for Subliminally and Supraliminally Presented Human Figures}

As discussed, the stimuli typically used in subliminal mere exposure studies are far removed from the complex, integrated stimuli generally encountered in daily life. Because the purpose of psychology is to understand nonlaboratory behavior, however, our experimental paradigms should approximate those situations that people encounter in daily life (Bornstein \& Masling, 1985). The second experiment tested the hypothesis that mere exposure effects similar to those obtained in Experiment 1 are obtainable with complex human figures. Undergraduate subjects were exposed to a series of photographs of men and women between the ages of 40 and 65 , with exposure duration preset at either 4-ms, 48-ms, or 200-ms. We hypothesized a significant interaction between choice type (affect vs. recognition) and exposure duration; whereas recognition accuracy should rise from chance level at 4-ms to almost $100 \%$ accuracy at $200 \mathrm{~ms}$, the accuracy of affect preferences should be signifcantly greater than chance at all exposure times but should not increase significantly with increasing exposure duration (see, e.g., Seamon et al., 1984).

It is possible that our procedure of collecting all affect ratings before obtaining recognition ratings on the same stimuli might have attenuated recognition accuracy in Experiment 1, because subjects were sometimes exposed to both stimuli from a given pair before making a recognition judgment. Therefore, in Experiment 2 the effect of order of judgments (i.e., affect first vs. recognition first) was examined directly. In addition, two different sets of photo stimuli were used in Experiment 2-one set for affect ratings and one set for recognition ratings-and stimulus-set order (i.e., Set 1 first vs. Set 2 first) was treated as an independent variable in this experiment.

\section{Method}

Subjects. Subjects were 100 undergraduate students ( 45 male and 55 female) enrolled in introductory psychology courses at SUNY Buffalo.
An additional 10 students (6 male and 4 female) participated only in the stimulus discrimination task.

Materials and apparatus. Stimuli were 24 photographs of human figures (6 male and 6 female photographs for Stimulus Set 1 and 6 male and 6 female photographs for Stimulus Set 2). Individuals photographed ranged from 40 to 65 years of age and were selected for use as stimuli on the basis of the following criteria: They fell within the normal range on pilot subjects' ratings of attractiveness, and they were not associated with the university and were therefore unlikely to be acquainted with any of the subjects in the experiment. Photographs were designed to be as similar as possible except for the facial features of the individuals pictured. Facial expressions of all photograph stimulus figures were neutral (i.e., neither smiling nor frowning). A common background (i.e., a white wall) was used in each picture, with lighting as similar across photos as possible. All photographs included only the individual's face and shoulders, in full frontal view, and in all cases the figures occupied approximately $35 \%$ of the total area of the picture. Photograph stimulus cards were constructed by using a procedure similar to that of Experiment 1: $3 \times 5$-in. photographs were glued on $5 \times 8$-in. white cardboard cards, yielding six pairs of male stimuli and six pairs of female stimuli.

Slide stimuli were constructed by having each photograph made into a standard $2 \times 2$-in. slide. The figures occupied approximately $20 \%$ of the total area of each slide. Stimuli were projected through a Scientific Prototype three-field tachistoscope, as in Experiment 1, with exposure duration, background field luminescence, and stimulus field luminescence preset by an experimenter who had no contact with the subjects. The experimenter who ran subjects was blind to all information regarding exposure duration and stimulus condition.

Procedure. The procedures used in Experiment 2 followed exactly the methodology described in Experiment 1, with the following exceptions. First, a $200-\mathrm{ms}$ condition was included because pilot testing revealed that subjects were often unable to make accurate recognition judgments of photographs presented for 48-ms, and we felt that in order to stay in parallel with Experiment 1, an exposure duration at which subjects could make very accurate recognition judgments was an important part of this experiment. Second, the illumination of the blank field remained at $15 \mathrm{fl}$, but the stimulus field illumination was $11 \mathrm{fl}$ throughout Experiment 2. Third, two sets of 6 stimulus pairs (rather than a total of 10 pairs, as in Experiment 1) were used in this experiment. Recognition ratings were made on different photographs than those used in the affect phase of the study. Finally, order of stimulus presentation (Stimulus Set 1 first vs. Stimulus Set 2 first) and judgment order (affect first vs. recognition first) were treated as independent variables and were counterbalanced across subjects.

Discrimination task. Ten additional subjects (6 male and 4 female) participated in a discrimination task to ascertain whether perception of the subliminally presented photos actually took place without awareness. The procedure of the task was identical to that described in Experiment 1 , except that photo stimuli were used in place of polygons.

\section{Results}

This experiment used a mixed design, with exposure duration (4-ms vs. 48-ms vs. 200-ms), stimulus order (Set 1 first vs. Set 2 first), and order of judgment (affect vs. recognition first) as between-subject variables and judgment type (affect vs. recognition) as a within-subject variable. No significant main effects or interactions were found for the variables of stimulus order and order of judgment; these dimensions have been dropped from subsequent analyses.

Percentage of previously seen stimulus selection as a function of judgment type and exposure duration is presented in Figure 


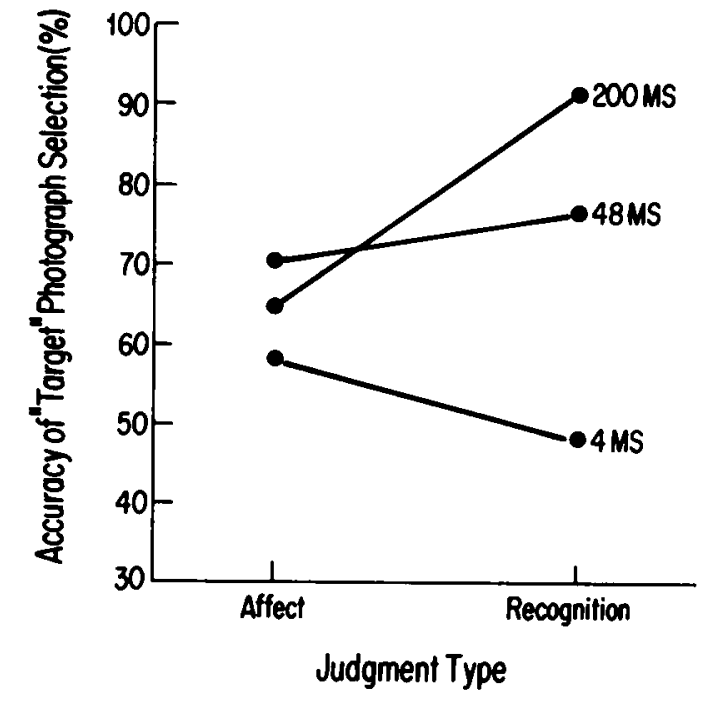

Figtre 2. Percentage of target (i.e., previously seen) photograph selection as a function of exposure duration ( $4 \mathrm{~ms}$ vs. $48 \mathrm{~ms} v \mathrm{vs} .200 \mathrm{~ms}$ ) and judgment type (affect vs. recognition).

2. Percentages of previously seen photograph selection in the affect and recognition conditions were, respectively, $58.1 \%$ and $48.1 \%$ for 4 -ms exposures; at $48-\mathrm{ms}$, these percentages were $70.5 \%$ and $76.0 \%$; at $200 \mathrm{~ms}$, they were $65.0 \%$ and $92.4 \%$.

Analysis of variance showed significant main effects for exposure duration, $F(2,97)=24.27, p=.001$, and judgment type, $F(1,97)=6.05, p=.02$, and a significant interaction between these variables, $F(2,97)=22.17, p=.001$. A $t$ test comparing the two types of judgments in the 4-ms condition showed that affect judgments were significantly more accurate than recognition judgments, $t(66)=3.51, p=.001$. Affect judgments in the 4-ms condition were also significantly more accurate in selecting previously exposed stimuli than would be expected by chance, $\chi^{2}(1, N=34)=26.24, p<.001$.

The mean number of slides correctly identified by subjects in the discrimination task was $30.9(52 \%)$. Overall, subjects correctly identified 309 of 600 slides, which does not differ significantly from chance accuracy, $\chi^{2}(1, N=600)=0.54$. The number of correct identifications ranged from $23(38 \%)$ to $35(58 \%)$.

\section{Discussion}

The results of Experiment 2 support the hypothesis that repeated subliminal presentation of photographs of human figures significantly enhances subjects' attitudes toward these photograph figures. This suggests that attitudes toward complex stimuli are affected by repeated subliminal exposures in a manner similar to the exposure effects obtained with simpler, less affect-laden stimuli such as polygons. Even when the accuracy of recognition judgments-that is, objective familiarity-remained at chance levels, preference ratings of subliminally exposed and novel stimuli were significantly different, with the previously exposed stimuli receiving more positive ratings. The results of the stimulus discrimination task used in Experiment 2 further indicate that subjects did not perceive consciously any aspects of the subliminally presented stimuli; subjects achieved only chance performance on this task. It seems then that subliminal presentation of photographs of human figures signifcantly influences subjects' attitudes toward the figures, producing typical mere exposure effects. These findings are consistent with those obtained by Kunst-Wilson and Zajonc (1980), by Seamon et al. (1984), and in our first experiment using simpler, neutral stimuli.

Interestingly, although we found no significant increase in affect ratings with increasing exposure duration in Experiment 1 , a significant increase in these ratings with increasing exposure duration was obtained in Experiment 2. This result is most likely due to differences between the photograph and polygon stimuli. It may be that familiarity with human faces is somehow more rewarding than is familiarity with geometric figures, resulting in significantly more positive affect ratings for faces with increasing familiarity. Alternatively, complexity of the stimuli may be a significant factor; a review of mere exposure studies conducted during the past 20 years suggests that complex stimuli generally produce stronger mere exposure effects than do simple stimuli (Bornstein, 1987; see also Saegert \& Jellison, 1970). In that context, perhaps the relatively complex photo stimuli used in this study resulted in increasingly positive affect ratings with increasing exposure duration, whereas the similar polygon stimuli produced a ceiling in affect ratings before a 48ms exposure duration was reached.

\section{Experiment 3-Subliminal Stimulation in Vivo: The Effects of Prior Subliminal Exposure on Perceptions of and Interactions With Others}

Whereas Experiment 2 investigated subjects' responses to subliminal presentation of photographs of human faces, the purpose of this experiment is to determine the extent to which such exposure influences subjects' perceptions of and interactions with people encountered in the natural environment of the psychology experiment. This experiment investigates the hypothesis (derived in part from the writings of Zajonc, 1968, 1980) that subjects' in vivo perceptions of other people can be systematically and predictably influenced by subliminal exposure to an image of the other person. We hypothesized that subliminal exposure to the image of a person would increase positive affect toward that person, even when the subject is unaware that exposure has occurred. In this experiment, subjects participated in an audiotaped triadic interaction with two confederate "subjects" involving discussion of a neutral topic (i.e., the gender of several unknown poets) on which the confederates were instructed to disagree, thereby placing the naive subject in the position of tiebreaker. Before interacting with the confederates, each subject was exposed subliminally either to a photograph of one of the confederates or to a blank slide. Following the interaction, the subject completed a postsession questionnaire on which he made several ratings of each confederate. Audiotapes were analyzed by using Bales's (1950) interaction process analysis scoring system to evaluate the quality of interaction between the subject and each confederate.

This experiment used a between-subjects design with stimulus type (a photograph of Confederate 1, a photograph of Confederate 2 , or a blank slide) as the between-subjects variable. 
Three dependent measures were used: the proportion of poems in which the subject concurred with the opinion of the previously exposed confederate, the quality of verbal communication directed to the target and control confederates, and the subject's postsession ratings of each confederate. The hypotheses of the experiment were as follows: that subjects will concur more often with the opinion of the previously seen confederate than with the opinion of the other triad member; that the subject will make more positive statements to, ask more questions of, and interact more frequently with the target than with the control confederate; and that the subjects' postsession ratings of the target confederate will be significantly more positive than will ratings of the control confederate.

\section{Method}

Subjects. Subjects were 64 male undergraduates enrolled in introductory psychology classes at SUNY Buffalo. An additional 10 male undergraduate subjects participated in a stimulus discrimination task similar to that used in Experiments 1 and 2.

Materials and apparatus. Stimuli were a blank slide and two standard $(2 \times 2$ in.) slides made from photographs of undergraduate male research assistants who served as confederate subjects. Slides were as similar as possible except for the facial features of the individual pictured, and both stimulus figures were photographed with neutral facial expressions. Each stimulus slide included only the figure's face and shoulders in full frontal view, and in both cases the figure occupied approximately $25 \%$ of the total area of the slide. As in Experiments 1 and 2, stimuli were presented through a Scientific Prototype three-field tachistoscope, with parameters preset by an experimenter who had no contact with the subjects. The experimenter who ran subjects was blind to all information regarding stimulus condition.

Procedure. Each subject was told that he was participating in a study of the decision-making process. He was informed that, along with the other two "subjects" in the experiment, he would be asked to read a series of 10 poems, following which the three of them should try to come to a consensus decision regarding the gender of each poet during a 10-min discussion period. Previous research has shown that this task is sufficiently interesting to keep the subject's attention and maintain his investment in the study and that subjects achieve chance accuracy on this task (Bornstein, Masling, \& Poynton, 1987). The confederates were instructed to disagree with each other regarding 7 of the 10 poems, placing the actual subject in the position of being the tiebreaker in each case. Activity of the confederates (i.e., speaking first, choice of gender for a given poet) was counterbalanced across subjects. The discussion took place in a sparsely furnished room as isolated from noise and distraction as possible. The seating arrangement was predetermined by the experimenter and was designed to place the subject in a position equidistant from and with equal visual access to both confederates. Discussions were audiotaped with a cassette recorder placed in the center of the discussion table. Audiotapes of the interaction were retained for later analysis.

When the subject arrived at the laboratory, he was informed that the first task in the study was a priming procedure that involved viewing pictures of stimuli "related to the content of the poems" and that the other two subjects in the study had arrived early and had already gone through the procedure. The subliminal priming procedure actually consisted of five 4-ms exposures, $5 \mathrm{~s}$ apart, of either a blank slide or one of the two confederate subjects. The blank slide was a control condition by which subjects' baseline preference ratings for each confederate were ascertained. As in Experiments 1 and 2, a 4-s bright energy field mask with a focus dot in the center was presented prior to each 4-ms exposure.
Table 1

Study 3: Number of Subjects Agreeing with Confederate 1 Versus Confederate 2 in the Poem Task as a Function of Subliminal Priming Material

\begin{tabular}{ccc}
\hline & \multicolumn{2}{c}{ Number of subjects agreeing } \\
\cline { 2 - 3 } Contents of & Confederate I & Confederate 2 \\
\hline priming material & 15 & 6 \\
Confederate 1 & 7 & 13 \\
Confederate 2 & 10 & 11 \\
Blank slide & 10 & \\
\hline
\end{tabular}

Note. $N=62 ; 2$ subjects run under experimental conditions who agreed with each confederate on an equal number of poems have been dropped from the analysis.

Following the discussion, each subject was asked to complete a postsession questionnaire, which consisted of the questions and ratings summarized in Table 2, along with several other filler ratings. To maintain credibility, each confederate also completed a copy of the postsession rating forms. Following the completion of postsession ratings, the subject was debriefed and informed of the deception involved in the study and of the purposes of the study.

Analysis of audiotaped interactions. Two experimenters blind to experimental condition rated each statement made by the actual subject using Bales's (1950) 12-category scoring system. For each statement, the raters noted the person to whom the communication was directed (i.e., Confederate 1 or Confederate 2). Interrater reliability in Bales scoring (coefficient kappa; Spitzer, Cohen, Fliess, \& Endicott, 1967), calculated from a sample of 10 audiotapes containing a total of 391 statements made by the subject, was determined to be 76 .

Discrimination task. In the discrimination task, 10 male undergraduates who had not participated in earlier phases of this experiment were asked to discriminate target (i.e., figure) slides from blank slides under subliminal conditions. The procedure of this task was identical to that used in Experiments 1 and 2, except that stimuli consisted of photographs of the confederate subjects.

\section{Results}

Agreement with poem judgments of previously seen and novel confederate subjects. Because the discussions were limited to $10 \mathrm{~min}$, different triads discussed varying numbers of poems. For the purposes of analysis, agreement with one or the other confederate is defined as agreement with that person on the majority of poems discussed. A total of 43 triads was run under conditions in which the subject was subliminally exposed to either a photograph of Confederate 1 or a photograph of Confederate 2. Two of these discussions ended with the subject agreeing with each confederate on an equal number of poems. These two triads have been dropped from the present analysis, leaving a total of $\mathbf{4 1}$ subjects run under noncontrol conditions.

Subjects agreed with the target confederate on the majority of poems in 28 of 41 cases; in the remaining 13 cases, the subject concurred with the opinion of the nontarget confederate, $\chi^{2}(1$, $N=41)=6.24, p=.012$. A summary of subjects' overall agreement with each confederate as a function of the subliminal priming material is presented in Table 1 . Triads debated an average of $5.94(S D=1.20)$ poems, with subjects agreeing with the target confederate on an average of $3.39(S D=1.03)$ poems.

Bales analysis. Only those subjects who were subliminally 
exposed to a photograph of one of the confederates prior to the triadic interaction are included in this analysis; those exposed to a blank slide were used as control subjects to establish that baseline interaction with each confederate was comparable. We compared the number of statements in each Bales category made to target and nontarget confederate subjects by use of $t$ tests.

Subjects' frequency of responding to target and nontarget confederates was significantly different only for 1 of the 12 Bales categories; they made a significantly greater number of Category 6 statements ("gives suggestion") to target than to nontarget confederates, $t(42)=2.04, p=.05$. Subjects made an average of $3.43(S D=1.97)$ suggestions to the target confederate and an average of $1.74(S D=1.54)$ suggestions to the nontarget confederate.

Postsession ratings. A summary of subjects' postsession ratings of target and nontarget confederates is presented in Table 2. As Table 2 shows, subjects' ratings of target and nontarget confederates were not significantly different, although target confederates received more positive ratings than nontarget confederates in four of the five categories of responding.

Stimulus discrimination task. The mean number of slides correctly identified in the discrimination task was $31.1(52 \%)$. Overall, subjects correctly identified 311 of 600 total slides, which does not differ significantly from chance performance, $\chi^{2}(1, N=600)=0.81$. The number of correct identifications ranged from $28(46 \%)$ to $36(60 \%)$.

\section{Discussion}

The results of Experiment 3 suggest that behavior in a triadic decision-making task is significantly influenced by previous subliminal exposure to one of the other group members; subjects agreed with the opinion of the previously exposed triad member significantly more often than they agreed with the opinion of the unfamiliar person. Bales analysis of the audiotaped triadic interactions indicated, however, that subjects were interacting with target and nontarget triad members with approximately equal frequency and were making similar types of statements to each. Similarly, analysis of subjects' postsession ratings indicated that they viewed the two confederates as equally likable, as having contributed equally to the discussion, and as equally well-known as a result of the triadic interaction. Results of the stimulus discrimination task confirmed that subjects were unaware of the influence of the subliminal priming material.

Although these results indicate that subjects' interactions with the target and nontarget confederates were comparable, several alternative explanations of this finding are possible. It may be that the Bales analysis was inadequate to assess differences in verbal responding; some other method of analyzing verbal interactions might have yielded different results. It is also possible that subjects' nonverbal interactions with the target and nontarget confederates were significantly different; however, this was not assessed in the present analysis. Finally, it may be that the negative finding in this area is a function of the experimental task; although subjects' interactions with target and nontarget confederates in discussing the gender of poets were not significantly different, some other type of experimental task
Table 2

Postsession Ratings of Previously Seen and Novel Confederate Subjects

\begin{tabular}{|c|c|c|}
\hline \multirow[b]{2}{*}{ Rating } & \multicolumn{2}{|c|}{ Confederate subject } \\
\hline & $\begin{array}{c}\text { Previously } \\
\text { seen }\end{array}$ & Nove \\
\hline \multicolumn{3}{|l|}{ Contribution } \\
\hline$M$ & 6.47 & 6.38 \\
\hline$S D$ & 1.59 & 1.83 \\
\hline \multicolumn{3}{|l|}{ Liking } \\
\hline$M$ & 6.04 & $5.8 \mathrm{I}$ \\
\hline$S D$ & 1.84 & 1.57 \\
\hline $\begin{array}{l}\text { Which confederate subject got along } \\
\text { with better during the discussion }\end{array}$ & 54.1 & 45.9 \\
\hline & & \\
\hline know better during the discussion & 52.4 & 47.6 \\
\hline $\begin{array}{l}\text { Which confederate subject would } \\
\text { prefer to talk to more and get to } \\
\text { know better after the discussion }\end{array}$ & 48.1 & 51.9 \\
\hline
\end{tabular}

Note. Only those subjects run under conditions in which prior exposure to a confederate subject occurred are included in this table (i.e., only those subjects run under experimental conditions); $n=43$. Contribution and liking ratings were made on 9-point bipolar scales, with a higher number indicating a more positive rating. The other ratings were forced choice, with subjects required to report (for example) which confederate subject they "got along with better" during the discussion. These ratings are expressed as percentages in this table.

(e.g., "get to know the people in your group during the next 10 minutes") might have yielded different results.

With regard to subjects' postsession ratings, similar problems of interpretation arise. Though there may have been no real differences in subjects' perceptions of the target and nontarget confederates, it is also possible that this negative finding results from the particular items included in the postsession questionnaire. Other rating dimensions or a more open-ended rating system might have produced different results. Similarly, a different experimental task might have resulted in significant differences in subjects' reported perceptions of target and nontarget confederates.

\section{General Discussion}

These experiments indicate that the subliminal mere exposure effect originally obtained by Kunst-Wilson and Zajonc (1980) and Seamon et al. (1984) with simple polygon stimuli is robust and replicable (Experiment 1), generalizable to complex, socially relevant stimuli (Experiment 2 ), and may be elicited under both laboratory and naturalistic conditions (Experiments 2 and 3 ). In each experiment, subliminal stimulus exposure resulted in a significant enhancement in affect toward the subliminally exposed stimulus. Furthermore, two different measures of awareness (recognition and discrimination judgments) were used in these experiments to ascertain whether subjects were able consciously to perceive any aspects of the subliminally presented stimuli.

It is interesting that we consistently found more positive 
affect ratings with increasing number of exposures even though a massed exposure sequence was used for both polygon and photograph stimuli. This contrasts with the observation that massed stimulus presentation generally produces an attenuation of mere exposure effects and sometimes produces no exposure effect whatsoever (e.g., Harrison \& Crandall, 1972). In fact, a meta-analysis of mere exposure studies conducted between 1968 and 1987 showed that sequence of stimulus presentations (i.e., massed vs. distributed) was a significant factor in the strength of the observed exposure effect, with studies ising distributed presentations obtaining more positive results than studies using massed presentations (Bornstein, 1987). It may be that in these experiments the boredom that typically results from massed presentations was obviated by the brief stimulus exposure duration. Although the procedure of this study did involve five repetitions of a stimulus with no intervening stimuli presented, each presentation was, at most, $200 \mathrm{~ms}$. Thus, the maximum total exposure duration of a stimulus in this study was $1 \mathrm{~s}$, whereas most stimuli were exposed for a far briefer period (as little as $20 \mathrm{~ms}$ total for stimuli in the 4-ms condition). The relatively small number of exposures and very brief exposure duration probably worked against boredom's being a significant factor in this study, despite our using massed stimulus presentations.

Our findings suggest that decisions made in interpersonal and social situations may be based in part on subliminally or marginally perceived stimulus qualities. To some extent, social cognitions, interpersonal judgments, and object choices may be influenced by properties of stimulus objects of which we are unaware. Our results are consistent with those of Bargh and Pietromonaco (1982), who found that subliminal verbal stimuli significantly influence impressions of others about whom they have little or no previous information. However, questions remain regarding the extent to which our results (as well as those of Bargh \& Pietromonaco) are generalizable to actual social situations. In other words, are subliminal effects a significant variable influencing attitudes toward others, object choice (and object avoidance), and social cognitions and behaviors outside of the laboratory? The answer is probably yes, but the nature of in vivo subliminal effects differs significantly from those explored in the lab. Clearly, our feelings and attitudes regarding others are based in part on aspects of the person, or of our interaction with the person, that are not verbalizable but take the form of a gut feeling regarding the person that leads us to like or dislike them. For example, to the extent that someone reminds us of an important figure from our past, our attitude regarding that person is likely to be influenced by our internalized representation of the past figure. In this context, the links between attribution theory and object relations theory are clear. Whereas attribution theory suggests that our perceptions of others in social situations are subjective, actively constructed, and sensitive to situational and interpersonal demands, psychoanalytic object relations theory represents an individual differences approach to the same issue, arguing that our perceptions of others are not only actively constructed but are based largely on past relationships with significant others. For the object relations theorist, past relationships serve as blueprints onto which new, unfamiliar people are mapped (Bornstein, Galley, \& Leone, 1986). Similarly, transference in psychotherapy involves the attribution of qualities from past relationships onto a present relationship, unintentionally and unconsciously. These events are not subliminal in the classical sense of being inaccessible to awareness under the most stringent laboratory conditions, but they do represent examples of the influence of stimuli-or aspects of stimuli-whose most important influence on behavior takes place because of perceptions and processes that are largely unconscious. Obviously, there are many differences between these events and laboratory investigations of subliminal mere exposure effects as influences on object choice and attitudes toward others. Clearly, also, the heuristic value of subliminal perception research needs to be extended more fully to in vivo social and interpersonal situations. However, there are also marked similarities between laboratory investigations of subliminal phenomena and the role of these phenomena in actual social and interpersonal situations (e.g., in both cases, attitudes are influenced by properties of the stimulus of which we are unaware, or at any rate that we are unable to verbalize). Research should now be directed to investigating the parameters and limits of subliminal and marginal effects in laboratory versus nonlaboratory situations.

The mechanism underlying the production of subliminal (as well as supraliminal) mere exposure effects also remains elusive. Harrison (1968) and Berlyne (1970) have discussed two possible models for these phenomena, but neither adequately accounts for the finding that conscious awareness of stimuli is not a prerequisite for the production of typical exposure effects. Both models suggest that objective familiarity with the stimulus is central in the elicitation of such effects. However, this notion is in conflict not only with the results of this study but also with past research on social judgment (Nisbett \& Wilson, 1977), with personality-based subliminal perception research (e.g., Silverman, 1983; Spence, 1964), and with models of selective attention and information processing (e.g., Triesman, 1969).

Zajonc (1980) has offered one speculative model for the mechanisms underlying subliminal mere exposure effects. Suggesting that "the assumption that component affect, utilities, or values attach themselves to the very same features that the subject attends to in a typical detection, recognition, discrimination, or categorization task is likely to be wrong" (Zajonc, 1980, p. 159; italics in original), Zajonc argued that affect and recognition judgments are based on different properties of a stimulus. He hypothesized that recognition judgments are based on specific, discrete features of the stimulus (i.e., size, shape, coloring) and referred to these features as discriminada, which he contrasted with preferenda: qualities of the stimulus on which affect judgments are based. Zajonc (1980) wrote,

I cannot be very specific about preferenda. If they exist they must be constituted of interactions between some gross object features and internal states of the individual-states that can be altered while the object remains unchanged, as, for example, when liking for a stimulus increases with repeated experience. (p. 159)

Zajonc (1984) later elaborated on this model, suggesting possible neuroanatomical pathways for affective responding in the absence of stimulus recognition (cf. Lazarus, 1984).

Zajonc's (1980) preferenda-discriminada model, although consistent with past research as well as our findings, is somewhat problematic. Criticisms of this model have been discussed 
elsewhere (e.g., Seamon et al., 1983a) and need not be reviewed here. However, some problems inherent in Zajonc's (1980) argument that are germane to the present issues warrant discussion. First, the basic concepts of Zajonc's model-preferenda and discriminada - are somewhat vague and are not operationally defined in such a way that properties of these two sets of features may be compared and evaluated. In addition, the means by which preferenda hold affect remains unclear, as is the mechanism underlying the observed positive attitude change in response to repeated stimulus exposures. Finally, Zajonc did not specify how stimuli such as words or nonsense syllableswhich are frequently used in studies of mere exposure effectsmay have preferendalike properties. Although it is easy to understand how, for example, the gestalt of a human face may have such properties, it is more difficult to conceive of words as having the global features characteristic of preferenda.

An alternative model of subliminal mere exposure effects based on psychodynamic principles and concepts may also be useful in understanding the mechanisms underlying this phenomenon. Though such a model presents certain difficulties, it is in many ways consistent with Zajonc's (1980) hypothesis yet represents a very different theoretical approach to the issue. Furthermore, no researchers or theoreticians in this field have discussed such effects from a psychoanalytic perspective.

Freud $(1905,1923)$ hypothesized that the ego seeks to refind new need-gratifying objects rather than search out new and unknown objects in an attempt to gratify needs. Thus, Freud (1923) wrote, "the first and immediate aim . . . of reality testing is not to find an object in real perception which corresponds to the one desired, but to refind such an object, to convince oneself that it is still there" (p. 238; italics in original). In short, the psychoanalytic perspective suggests that objects with which we have had pleasurable experiences are preferable to new objects about which we have relatively little data and no past experience.

Freud's (1905, 1923) model further suggests that a mental image, or representation, of objects with which we have had experience must be stored in memory, perhaps consciously or perhaps at a preconscious or unconscious level (see Bemporad, 1980 , for a discussion of the object representation concept in psychoanalysis). When minimal data regarding an unfamiliar object or stimulus are available, then, the new object is compared with representations of older, internalized objects, and decisions regarding the new object are made on the basis of past experience (although the representation of this experience may be unconscious). In terms of sheer probability, then, Freud's model implies that when confronted with choosing between an object that is somehow like mental representations of past objects and a new object that is entirely unfamiliar, the individual will tend to select the more familiar object, even though the person may not be able to articulate the basis for this choice (recalling Zajonc's notion of preferenda as the basis for affective object choice).

Taking this psychoanalytic perspective a step further, it is clear that object permanence (Piaget, 1937) is a prerequisite for the production of both subliminal and supraliminal mere exposure effects. If the individual does not have mental representations of past objects available to compare with new objects, then positive past experiences can have no influence on affect judg- ments of new, unfamiliar objects. A lack of object permanence should result in an attenuation of mere exposure effects.

The psychoanalytic model, with its emphasis on object permanence and the presence of internalized objects as necessary preconditions for the production of exposure effects, suggests several potentially valuable avenues of research. For example, mere exposure studies using very young subjects may help illuminate the role of object permanence in these phenomena, as would investigations of subjects who have lost the capacity to retain the mental image of an absent object (e.g., patients with certain organic brain syndromes). In addition, studies of driverelated stimuli (Silverman, 1983) may afford some insight into the relation of drive/need states to the production of exposure effects. In this area, it may be interesting to examine the relation of parental representations to exposure effects obtained by using pictures of older adults, authority figures, maternal figures, and so forth. It would also be useful to study exposure effects in clinical populations, whose members presumably have internalized objects of a different quality (e.g., more threatening, less nurturing, less consistent) than do members of nonclinical groups (see Blatt, 1974, for a discussion of the relation of object representations and psychopathology).

A number of other issues relevant to future research on subliminal mere exposure effects emerge from our findings. As discussed, probing the nature of the attitude change toward merely exposed stimuli may illuminate the mechanisms underlying mere exposure effects. It would also be interesting to examine the duration of this attitude change over time. Certain studies (e.g., Seamon et al., 1983b) suggest that subliminal exposure effects are stable over extended periods. It may now be useful to examine how attitudes toward subliminally exposed stimuli change over time, how the nature of the stimulus (e.g., words vs. people, etc.) influences this temporal stability, and to what extent typical subliminal mere exposure effects may be obtainable long after stimulus exposure (e.g., 6 months following exposure) under both laboratory and naturalistic conditions. Examining the effects of repeated subliminal exposure to human figures that are nonneutral (e.g., figures that appear angry or frightened rather than being depicted with neutral facial expressions) may also prove to be a fruitful avenue for future research.

\section{References}

Bales, R. F. (1950). Interaction process analysis. Reading, MA: Addison-Wesley.

Bargh, J. A., \& Pietromonaco, P. (1982). Automatic information processing and social perception: The influence of trait information presented outside of conscious awareness on impression formation. Journal of Personality and Social Psychology, 43, 437-449.

Bemporad, J. R. (1980). Review of object relations theory in light of cognitive development. Journal of the American Academy of Psychoanalysis, 8, 57-75.

Berlyne, D. E. (1970). Novelty, complexity and hedonic value. Perception and Psychophysics, 8, 279-286.

Blatt, S. J. (1974). Levels of object representation in anaclitic and introjective depression. Psychoanalytic Study of the Child, 29, 107-157.

Bornstein, R. F. (1987). Exposure and affect: Overview and meta-analysis of research, 1968-1987. Manuscript submitted for publication.

Bornstein, R. F., Galley, D. J., \& Leone, D. R. (1986). Parental representations and orality. Journal of Personality Assessment, 50, 80-89. 
Bornstein, R. F., \& Masling, J. M. (1984). Subliminal psychodynamic stimulation: Implications for psychoanalytic theory and therapy. International Forum for Psychoanalysis, 1, 187-204.

Bornstein, R. F., \& Masling, J. M. (1985). Orality and latency of volunteering to serve as experimental subjects. Journal of Personality Assessment, 49, 306-310.

Bornstein, R. F., Masling, J. M., \& Poynton, F. G. (1987). Orality as a factor in interpersonal yielding. Psychoanalytic Psychology; 4, 161170.

Dixon, N. F. (1981). Preconscious processing. New York: Wiley.

Freud, S. (1905). Three essays on the theory of sexuality. Standard Edition VII. London: Hogarth.

Freud, S. (1919). The interpretation of dreams. Standard Edil.un IV \& $V$. London: Hogarth. (Original work published 1900)

Freud, S. (1923). The ego and the id. Standard Edition XIX. London: Hogarth.

Green, D. M., \& Swets, J. A. (1966). Signal detection theory and psychophysics. New York: Wiley.

Harrison, A. A. (1968). Response competition, frequency, expectancy behavior and liking. Journal of Personality and Social Psychology, 9, 363-368.

Harrison, A. A., \& Crandall, R. (1972). Heterogeneity and homogeneity of exposure sequences and the attitudinal effects of exposure. Journal of Personality and Social Psychology, 21, 234-238.

Kunst-Wilson, W. R., \& Zajonc, R. B. (1980). Affective discrimination of stimuli that cannot be recognized. Science, 207, 557-558.

Lazarus, R.S. (1984). On the primacy of cognition. American Psychologist, 39, 124-129.

Masling, J. M., Bornstein, R. F., Poynton, F. G., Reed, S. D., \& Katkin, E. S. (1987). Subliminal stimulation and electrodermal responding. Manuscript submitted for publication.

McConnell, J. V., Cutler, R. L., \& McNeil, E. B. (1958). Subliminal stimulation: An overview. American Psychologist, 13, 229-242.

Nisbett, R. E., \& Wilson, T. D. (1977). Telling more than we can know: Verbal reports on mental processes. Psychological Review, 84, 231259.

Piaget, J. (1937). The construction of reality in the child. New York: Basic Books.

Poetzl, O. (1917). The relationship between experimentally induced dream images and indirect vision [Monograph No. 7]. Psychological Issues, 2, 41-120.

Saegert, S. C., \& Jellison, J. M. (1970). Effects of initial level of response competition and frequency of exposure on liking and exploratory behavior. Journal of Personality and Social Psychology, 16, 553-558.

Seamon, J. G., Brody, N., \& Kauff, D. M. (1983a). Affective discrimination of stimuli that are not recognized: Effects of shadowing, masking, and cerebral laterality. Journal of Experimental Psychology: Learning, Memory, and Cognition, 9. 544-555.

Seamon, J. G., Brody, N., \& Kauff, D. M. (1983b). Affective discrimination of stimuli that are not recognized: II. Effect of delay between study and test. Bulletin of the Psychometric Society, 21, 187-189.

Seamon, J. G., Marsh, R. L., \& Brody, N. (1984). Critical importance of exposure duration for affective discrimination of stimuli that are not recognized. Journal of Experimental Psychology: Learning, Memory, and Cognition. 10, 465-469.

Silverman, L. H. (1966). A technique for the study of psychodynamic relationships: The effects of subliminally-presented aggressive stimuli on the production of pathological thinking in a schizophrenic population. Journal of Consulting Psychology, 30, 103-111.

Silverman, L. H. (1977). Ethical considerations and guidelines in the use of subliminal psychodynamic activation. Unpublished manuscript, New York University, Research Center for Mental Health.

Silverman, L. H. (1983). The subliminal psychodynamic activation method: Overview and comprehensive listing of studies. In J. Masling (Ed.), Empirical studies of psychoanalytic theories (pp. 69-100). Hillsdale, NJ: Erlbaum.

Spence, D. P. (1964). Conscious and preconscious influences on recall. Journal of Abnormal and Social Psychology, 68, 92-99.

Spitzer, R. L., Cohen, J., Fliess, J. L., \& Endicott, J. (1967). Quantification of agreement in psychiatric diagnosis. Archives of General Psychiatry, 17, 83-87.

Triesman, A. M. (1969). Strategies and models of selective attention. Psychological Review; 76, 282-299.

Vanderplas, J. M., \& Garvin, E. A. (1959). The association value of random shapes. Journal of Experimental Psychology, 57, 147-154.

Winson, J. (1984). Brain and psyche: The biology of the unconscious. New York: Doubleday.

Zajonc, R. B. (1968). Attitudinal effects of mere exposure. Joumal of Personality and Social Psychology Monograph, 9 (2, Pt. 2), 1-27.

Zajonc, R. B. (1980). Feeling and thinking: Preferences need no inferences. American Psychologist, 35, 151-175.

Zajonc, R. B. (1984). On the primacy of affect. American Psychologist, $39,117-123$.

Received November 28, 1986

Revision received June 18,1987

Accepted April 30, 1987

\title{
Kintsch Appointed Editor of Psychological Review, 1989-1994
}

The Publications and Communications Board of the American Psychological Association announces the appointment of Walter Kintsch, University of Colorado, as editor of Psychological Review for a 6-year term beginning in 1989. As of January 1, 1988, manuscripts should be directed to

\author{
Walter Kintsch \\ Department of Psychology \\ University of Colorado \\ Campus Box 345 \\ Boulder, Colorado 80309
}

Manuscript submission patterns for Psychological Review make the precise date of completion of the 1988 volume uncertain. The current editor, Martin Hoffman, will receive and consider manuscripts until December 31, 1987. Should the 1988 volume be completed before that date, manuscripts will be redirected to Kintsch for consideration in the 1989 volume. 